\title{
Analysis of Alprostadil Injection in Real-World Usage and a Review of Literatures
}

Jingchao Yan ( $\nabla_{\text {jingchao.yan@fdeent.org })}$

Eye Ear Nose Throat Hospital of Fudan University

Jianwen Shen

Eye Ear Nose Throat Hospital of Fudan University

Ling Li

Eye Ear Nose Throat Hospital of Fudan University

Taomin Huang

Eye Ear Nose Throat Hospital of Fudan University

\section{Research Article}

Keywords: alprostadil injection, prescriptions, otolaryngology outpatients, utilization review

Posted Date: April 8th, 2021

DOI: https://doi.org/10.21203/rs.3.rs-374909/v1

License: (9) This work is licensed under a Creative Commons Attribution 4.0 International License. Read Full License 


\section{Abstract}

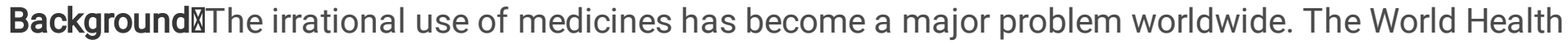
Organization (WHO) estimates that more than half of all medicines are prescribed, dispensed, or sold inappropriately, and advised the pursuit of the responsible use of medicines.In China regulations require that a pharmacist must audit the prescription.

Alprostadil injection is a vasodilator and an inhibitor of platelet aggregation There is no obvious or clear indication for its use in the ear, nose and throat (ENT) Department. It came to our notice that in our hospital, alprostadil injection has been prescribed in the ENT Outpatient Department to treat some diseases.

Aim: This utilization review on the use of alprostadil injection was carried out in the ear, nose and throat (ENT) Outpatient Department of our hospital to determine its utilization patterns and promote its rational use.

Methods: A prescription survey was conducted, and detailed demographic information, including the patient's age, gender, diagnosis and so on, were recorded in an Excel spreadsheet. The rationality for these prescriptions were retrospectively evaluated.

Results: Based on the evaluation results, all alprostadil injections were found to be used off label in the Otolaryngology Department. Furthermore, $98.3 \%$ of the prescriptions were for the treatment of sudden hearing loss, $1.1 \%$ of the prescriptions for the diagnosis of hypoacusis were considered empirical and should have been more specific, and $0.6 \%$ of the prescriptions were for the treatment of Ménière's disease and large vestibular aqueduct syndrome. There were various dosage regimens for alprostadil injection in clinical practice. However, there was no statistically significant difference in dosage between the different age groups. Moreover, most of the alprostadil injections were given by $I V$ bolus, while $12.2 \%$ of patients received this by intravenous drip.

Conclusion: The present review indicated that improvements should be made in the quality of prescriptions for alprostadil injection in the Otolaryngology Outpatient Department. Furthermore, there is no strong evidence to support the use of high doses. Future audits of such use may be combined with an awareness campaign to reduce and discourage the irrational use of off label medications.

\section{Introduction}

The irrational use of medicines has become a major problem worldwide. The World Health Organization (WHO) estimates that more than half of all medicines are prescribed, dispensed, or sold inappropriately [1], and advised the pursuit of the responsible use of medicines [1]. With the deepening of the Chinese healthcare reform, commissions of health and family planning at different levels are promoting the transformation of pharmaceutical care in China, and prescription review and comment has been emphasized [2]. Regulations require that a pharmacist must audit the prescription [2, 3]. If the medication 
is not appropriate, the pharmacist should inform the physician to confirm or modify the prescription. In the absence of vigilance by pharmacists regarding unreasonable prescriptions, academic medical institutions should take measures, such as education, training, criticism and so on, to warn the pharmacist concerned [3]. Therefore, it is important for pharmacists to understand the utilization patterns of drugs.

As a vasodilator and an inhibitor of platelet aggregation, alprostadil injection has been indicated in China for (1) the improvement of ulcers of the extremities and pain at rest associated with chronic arterial occlusion (thrombotic thrombovasculitis obliterans and atherosclerosis obliterans), and cerebral vascular microcirculation disorders, (2) antithrombotic therapy after organ transplantation, where it is used to inhibit thrombus formation in blood vessels after transplantation, (3) arterial catheter dependence in congenital heart disease, where it is used to relieve hypoxemia and maintain tube flow, while awaiting surgical treatment, and (4) adjuvant treatment of chronic hepatitis. There is no obvious or clear indication for its use in the ear, nose and throat (ENT) Department. It came to our notice that in our hospital, alprostadil injection has been prescribed in the ENT Outpatient Department to treat some diseases. Furthermore, few articles [4-6] have focused on the off label use of alprostadil in ENT patients. In order to understand its utilization patterns a survey of outpatient alprostadil injection prescriptions was performed to provide a prescription audit reference to the pharmacist, and promote the reasonable use of this medicine.

\section{Materials And Methods}

The present study was conducted in the ENT Outpatient Department of Fudan University Hospital, where approximately 1,500 patients are checked daily for ENT-related diseases. The pharmacy has only one product of alprostadil injection (Kaishi ${ }^{\mathrm{T}}$; Jiangsu Hengrui Medicine Co., Ltd, China). Ethics approval was obtained for the present study.

A survey of electronic prescriptions containing alprostadil injection for ENT outpatients was performed from January 2017 to December 2017. First, the detailed information about each prescription, including the patient's age, gender, diagnosis and dosage regimen and other information, were recorded in Excel spreadsheets. SPSS 15.0 was used for statistical analysis of the data. Then, the indication for the prescription was retrospectively evaluated. Furthermore, a relevant literature search was conducted to collect any information that supports the use of alprostadil injection.

\section{Results}

There were 752 ENT patients with alprostadil injection prescriptions in 2017. The age of these patients ranged within 5-88 years old, with a mean age of 46.9 years old. Among these patients, 371 patients were male, while 381 patients were female (Table 1). 
Table 1

Characteristics of the study population

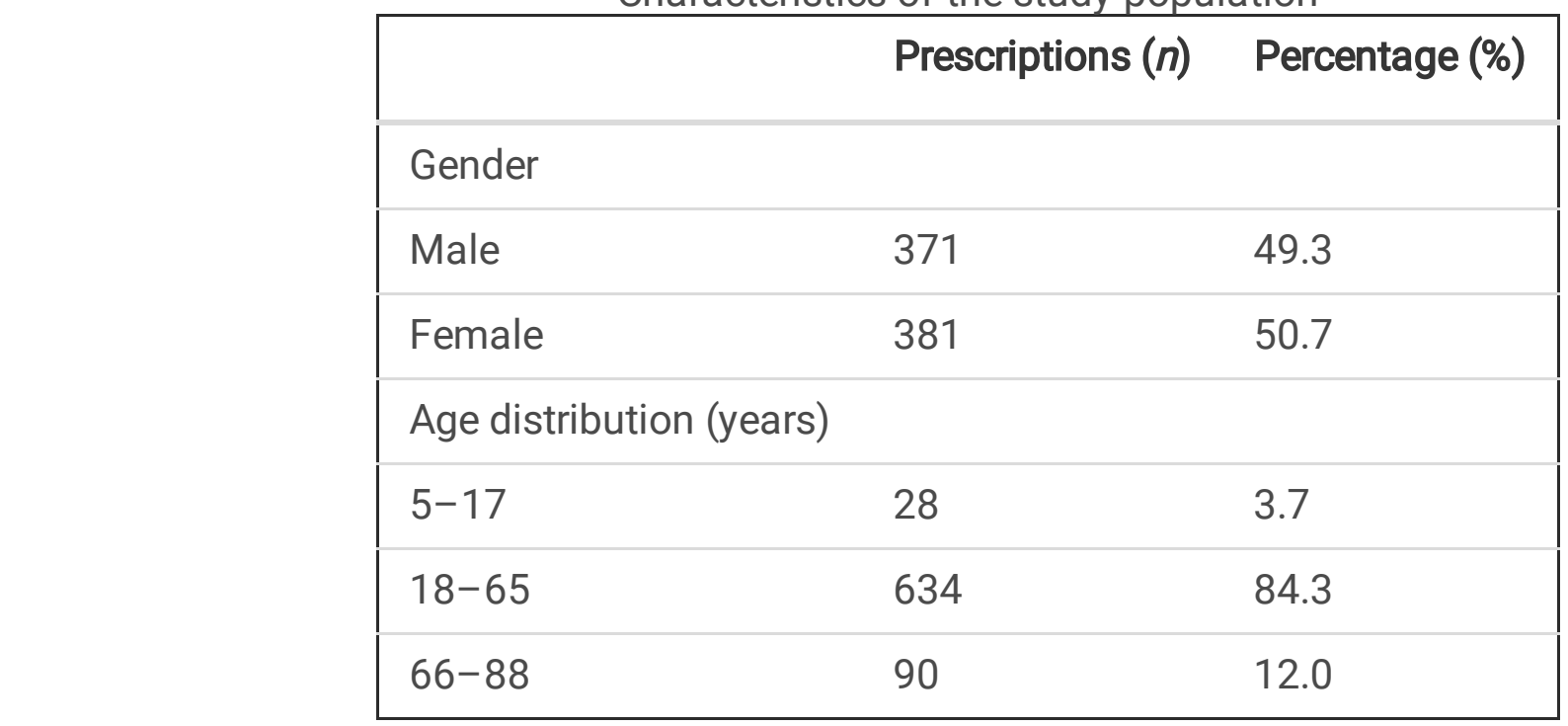

\section{Patient diagnosis}

For patients who had prescriptions for alprostadil injection, the diagnoses are summarized in Table 2 . Alprostadil injection was mainly used for the treatment of sudden hearing loss, but there were also few prescriptions for the treatment of hypoacusis, large vestibular aqueduct syndrome and Ménière's disease (Table 2).

Table 2

Diagnoses of patients with alprostadil injection prescriptions

\begin{tabular}{|lll|}
\hline Diagnosis & Prescriptions $(\boldsymbol{n})$ & Percentage (\%) \\
\hline Sudden hearing loss (SHL) & 739 & 98.3 \\
\hline Hypoacusis & 8 & 1.1 \\
\hline Large vestibular aqueduct syndrome (LVAS) & 3 & 0.4 \\
\hline Ménière's disease (MD) & 1 & 0.1 \\
\hline SHL \& LVAS & 1 & 0.1 \\
\hline
\end{tabular}

\section{Alprostadil Injection dosage regimen}

It was found that there are various dosage regimens for alprostadil injection, which ranged from $5 \mu \mathrm{gd}$ to $30 \mu \mathrm{g} q d$ ( Table 3). These patients were divided into three groups, according to age: $<18$ years old, $18-$ 65 years old, and $>65$ years old. One way ANOVA was performed on these three groups. No significant difference in dosage was found among the different age groups, but there were raising concerns about its inappropriate dosage. 
Table 3

Dosage regimens for the alprostadil injection

\begin{tabular}{|lll|}
\hline Dosage regimen & Prescriptions $(\boldsymbol{n})$ & Percentage (\%) \\
\hline $10 \mu \mathrm{g}, q d, i v$ & 658 & 87.5 \\
\hline $10 \mu \mathrm{g}, q d, i v g t t$ & 90 & 12.0 \\
\hline $7 \mu \mathrm{g}, q d, i v g t t$ & 1 & 0.1 \\
\hline $5 \mu \mathrm{g}, q d, i v g t t$ & 1 & 0.1 \\
\hline $20 \mu \mathrm{g}, q d, i v$ & 1 & 0.1 \\
\hline $30 \mu \mathrm{g}, q d, i v$ & 1 & 0.1 \\
\hline Total & 752 & 100.0 \\
\hline
\end{tabular}

Notes: qd, once a day; IV, intravenous injection; ivgtt, intravenous drip

Most of the prescriptions were for $I V$ bolus, while some were for intravenous drip.

The alprostadil injections were prescribed by 34 doctors.

\section{Discussion}

\section{Medication suitability}

There are no approved otorhinolaryngologic indications for alprostadil injection in China. All alprostadil injections prescribed in the Otolaryngology Department were off label. As observed in the present review, 98.3\% of alprostadil injections prescribed in the ENT Outpatient Department were for the treatment of sudden hearing loss. Microvascular dysfunction in the cochlea has been considered the major cause of sudden hearing loss $[7,8]$. As a vasodilator and an inhibitor of platelet aggregation, the injection of alprostadil has been considered to be effective in enhancing cochlear blood flow and oxygen supply. Hence, alprostadil has been used for the treatment of sudden hearing loss. The use of alprostadil for the treatment of sudden deafness is not the practice of individual doctors, but is commonly observed among otologists. According to the data of the Chinese multicenter clinical research on sudden deafness, drugs for improving inner ear microcirculation are effective for all types of sudden deafness [8]. The results of a meta-analysis conducted on 13 randomized studies suggested that patients treated with alprostadil presented with improved hearing, when compared with controls [7]. In the guideline for sudden hearing loss of China, drugs for enhancing inner ear microcirculation are recommended for use in high-frequency hearing loss, as well as in all frequency-related hearing loss [9]. However, the clinical practice guideline for sudden hearing loss in the USA is against the routine prescription of vasodilators or vasoactive substances for the treatment of sudden hearing loss [10]. Hence, differences exist on many issues in the guidelines for sudden deafness in various countries, which may be correlated to different medical modalities, insurance mechanisms and clinical experiences. 
Furthermore, $1.1 \%$ of the prescriptions for the diagnoses of hypoacusis were considered too broad and empirical, since there are many causes of hearing loss, such as infection, noise exposure and age [11]. In the absence of a clear indication by the prescribing doctor, it is difficult for the pharmacist to estimate the suitability of the drug.

The treatment protocols for Ménière's disease differ from one center to another, even in the same country, but the international consensus on its treatment does not recommend the use of vasodilators or alprostadil [12].

Large vestibular aqueduct syndrome is a congenital disorder, and there is no medical therapy available to treat the fluctuating or progressive sensorineural hearing loss [13]. Large vestibular aqueduct syndrome causing sudden sensorineural hearing loss can be treated the same way as sudden hearing loss [14]. According to present literatures, no sufficient evidence has proven that alprostadil is effective.

\section{Dosage regimen}

The recommended dosage by the drug description for alprostadil injection is within 5-10 $\mu \mathrm{g}$. It was found that there are various dosage regimens for alprostadil injection worldwide, and $10 \mu \mathrm{g} q \mathrm{~d}$ was the most commonly used dose in both adult and pediatric practice. However, there was no statistical difference between doses in children and adults in the present study. Furthermore, there is no recommended dosage for alprostadil injection in children in existing literature. Hence, due attention should be given to children receiving adult doses for the early detection of adverse drug reactions. In the present study, two adult patients were given $20 \mu \mathrm{g}$ and $30 \mu \mathrm{g}$, respectively. Furthermore, several articles have reported a good therapeutic effect for the use of high doses of lipoprostaglandin E1 [15, 16], but these articles were all case reports. Hence, there is no strong evidence to support the use the high doses, which is thereby not recommended. The pharmacist should confirm such prescriptions with the physician regarding suitability.

\section{Intravenous drip or IV bolus}

Furthermore, $12.2 \%$ patients received alprostadil injection by intravenous drip. However, it should be administered through IV bolus or Murphy's dropper, according to the dosage recommendations for the drug. Alprostadil injection, also called Lipo-PGE1, is a new PGE1 with lipid microspheres as a drug carrier. It is an oil-in-water type micro emulsion. Theoretically, if it is diluted in too much water soluble solvent, it easily leads to de-emulsification, and PGE1 is released from the lipid particles. As a result, phlebitis can occur at the site of the venepuncture, and the lipoprostaglandin E1 may be less effective due to its short half-life. However, existing clinical application research results were contradictory. Some studies have suggested that the drug should be given by $I V$ bolus [17], while other studies suggested that $I V$ bolus and Murphy's dropper increase the incidence of phlebitis, and that intravenous drip is the best route of administration [18]. Although alprostadil injections were mostly administered by intravenous drip in our hospital, no adverse drug reactions were observed. Further research on the method of administration could provide a clear consensus on this issue. 


\section{Limitation of the study}

The present study was carried out in a single center and single department, thereby causing selection bias.

\section{Declarations}

Conflict of interest: The authors declare that there are no conflicts of interest with this work.

Ethics approval and consent to participate: All procedures performed in the present study that involved human participants were in accordance with the ethical standards of the institutional research committee, and the 1964 Helsinki declaration and its later amendments or comparable ethical standards. The Ethics Committee of Shanghai Eye, Ear, Nose and Throat Hospital, Shanghai Fudan University, Shanghai, China approved the present study. Informed consent was obtained from all subjects or, if subjects are under 18, from a parent and/or legal guardian.

Consent for publication』Written informed consent for publication was obtained from all participants.

Availability of data and materials[The datasets used or analysed during the current study are available from the corresponding author on reasonable request.

Competing interests[The authors declare that they have no competing financial interests.

Authors' contributions $\square$ Conception and design: jingchao Yan; Administrative support: taomin Huang; Provision of study materials: jingchao Yan jianwen Shen; ling Li; Data analysis and interpretation: jingchao Yan jianwen Shen

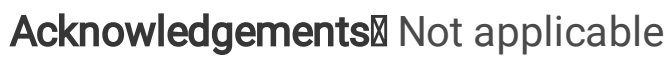

\section{References}

1. WHO, The Pursuit of Responsible Use of Medicines: Sharing and Learning from Country Experiences. http://www.who.int/medicines/areas/rational_use/en/ Accessed 29 Dec 2017.

2. Shanghai municipal commision of health and family planning(2017). Notice on the further strengthening of prescription review and prescription review in Shanghai and promoting rational use of medicine. http://www.wsjsw.gov.cn/wsj/n429/n432/n1487/n1509/u1ai140325.html\# Accessed 29 Dec 2017.

3. Shanghai municipal commision of health and family planning(2017). Notice concerning the issue of

" Administrative regulations on the prescription comments of Shanghai medical institutions". http://www.wsjsw.gov.cn/wsj/n429/n432/n2354/n2357/u1ai142052.html Accessed 29 Dec 2017.

4. Shim, H.S., J.S. Kim and M.G. Kim, Comparative Analysis of the Combined Therapeutic Effects of Lipoprostaglandin E1 on Sudden Idiopathic Sensorineural Hearing Loss. Journal of Audiology and 
Otology, 2017. 21(1): p. 33-38.

5. Lee, H.Y., et al., Therapeutic effect of combined steroid-lipoprostaglandin E1 for sudden hearing loss: a propensity score-matched analysis. American Journal of Otolaryngology, 2015. 36(1): p. 5256.

6. Jiang J, W.S.T.K., Synergic effect of alprostadil injection and ginaton in treating sudden deafness. Lin Chung Er Bi Yan Hou Tou Jing Wai Ke Za Zhi, 2011. 25(13): p. 597-599.

7. Zhuo, X.L., et al., Is the application of prostaglandin E1 effective for the treatment of sudden hearing loss? An evidence-based meta-analysis. J Int Med Res, 2008. 36(3): p. 467-70.

8. Surgery, E.B.O.C. and C.M.A. Society Of Otorhinolaryngology Head And Neck Surgery, Guideline of diagnosis and treatment of sudden deafness (2015). Zhonghua Er Bi Yan Hou Tou Jing Wai Ke Za Zhi., 2015. 50(6): p. 443-447.

9. Editorial committee of Chinese journal of otorhinolaryngology head and neck surgery and Chinese medical association otolaryngology head and neck surgery branch. Guidelines for the diagnosis and treatment of sudden deafness (2015). Chinese journal of otolaryngology head and neck surgery, 2015(6): p443-447.

10. Stachler, R.J., et al., Clinical Practice Guideline: Sudden Hearing Loss. Otolaryngology-Head and Neck Surgery, 2012. 146(3_suppl): p. S1-S35.

11. Lasak, J.M., et al., Hearing Loss. Primary Care: Clinics in Office Practice, 2014. 41(1): p. 19-31.

12. Nevoux, J., et al., International consensus (ICON) on treatment of Ménière's disease. European Annals of Otorhinolaryngology, Head and Neck Diseases, 2018. 135(1): p. S29-S32.

13. Goh, E., et al., Familial enlarged vestibular aqueduct syndrome. American Journal of Otolaryngology, 2001. 22(4): p. 286-290.

14. Lee, S.S., et al., Fate of Sudden Deafness Occurring in the Only Hearing Ear: Outcomes and Timing to Consider Cochlear Implantation. Journal of Korean Medical Science, 2010. 25(2): p. 283.

15. Hong, L., J. Zhang and J. Shen, Clinical efficacy of different doses of lipo-prostaglandin E1 in the treatment of painful diabetic peripheral neuropathy.

16. Ogawa, K., et al., Effect of prostaglandin E1 on idiopathic sudden sensorineural hearing loss: a double-blinded clinical study. Otol Neurotol, 2002. 23(5): p. 665-8.

17. Chi $\mathrm{HQ}$, Investigation and analysis on the use of alprostadil injection in our hospital. Strait Pharmaceutical Journal, 2017,29(11): 264-267.

18. Ou JM, Li Y. Analysis of phlebitis caused by different infusion methods of prostol injection. Clinical medicine research and practice, 2016,1(15): 54. 